\title{
Device Electrical Evaluation Method
}

National Cancer Institute

\section{Source}

National Cancer Institute. Device Electrical Evaluation Method. NCI Thesaurus. Code C91919.

A test that evaluates the functioning of a device's electrical systems. 